\title{
Navigating Neocortical Neurogenesis and Neuronal Specification: A Positional Information System Encoded by Neurogenetic Gradients
}

\author{
Bernhard Suter, ${ }^{1}$ Richard S. Nowakowski, ${ }^{2}$ Pradeep G. Bhide, ${ }^{1}$ and Verne S. Caviness ${ }^{1}$ \\ ${ }^{1}$ Department of Neurology, Massachusetts General Hospital, Boston, Massachusetts 02114, and ${ }^{2}$ Department of Neuroscience and Cell Biology, University \\ of Medicine and Dentistry of New Jersey-Robert Wood Johnson Medical School, Piscataway, New Jersey 08854
}

\begin{abstract}
The projection neurons of the neocortex are produced in the pseudostratified ventricular epithelium (PVE) lining the embryonic lateral ventricles. Over a $7 \mathrm{~d}$ period in mouse, these neurons arise in an overlapping layer VI-to-II sequence and in an anterolateral to posteromedial gradient [the transverse neurogenetic gradient (TNG)]. At any time in the $7 \mathrm{~d}$ neurogenetic interval, a given PVE cell must know what class of precursor cell or neuron to form next. How this information is encoded in the PVE is not known. With comparative experiments in wild-type and double-transgenic mice, overexpressing the cell cycle inhibitor $\mathrm{p} 27^{\text {Kip } 1}$, we show that a gradient of expression of Lhx2 (inferred from its mRNA levels), a LIM homeodomain transcription factor, together with a gradient in duration of the $\mathrm{G}_{1}$ phase of the cell cycle $\left(\mathrm{T}_{\mathrm{G} 1}\right)$, are sufficient to specify a positional mapping system that informs the PVE cell what class of neuron to produce next. Lhx2 likely is representative of an entire class of transcription factors expressed along the TNG. This mapping system consisting of a combination of signals from two different sources is a novel perspective on the source of positional information for neuronal specification in the developing CNS.
\end{abstract}

Key words: positional information; neocortex; neurogenesis; cell cycle; Lhx2 expression; transverse neurogenetic gradient

\section{Introduction}

Neocortical projection neurons arise from a pseudostratified ventricular epithelium (PVE) within the ventricular zone (VZ) of the embryonic cerebral wall (Boulder Committee, 1970; Takahashi et al., 1995). The separate cortical laminar neuron classes arise in an overlapping layer VI-to-II "inside-out" succession in mice during 7 d involving 11 cell cycles (Takahashi et al., 1995; Miyama et al., 1997; Nowakowski et al., 2002). The generation of successive neuronal classes begins at the anterolateral boundary of the PVE and propagates posteromedially across the cerebral wall along the transverse neurogenetic gradient (TNG). As a consequence, at any given time the PVE cells at different locations along the TNG produce different laminar classes of neurons. This requires that a PVE cell must "know" at all moments "where" it is relative to other PVE cells and "when" it is in the laminar neuronal class specification sequence. How are these two components

Received March 13, 2007; revised Aug. 14, 2007; accepted Aug. 15, 2007.

This work was supported by United States Public Health Service Grants NS12005 (V.S.C.), NS43246 and DA20796 (P.G.B.), NS49445, and EY15647 and by a grant from the New Jersey Commission on Spinal Cord Research (R.S.N.). We are grateful to Dr. Charles Vanderburgh (Director of Advanced Tissue Resource Center, Harvard Center for Neurodegeneration and Repair, Massachusetts General Hospital) for advice and assistance on laser capture microdissection and quantitative real-time PCR and to Dr. Christian Waeber [Director of Microscopy and Image Analysis Core, Interdepartmental Neuroscience Center (P30NS045776), Massachusetts General Hospital] for advice and assistance with quantitative image analysis.

The authors declare no competing financial interests.

Correspondence should be addressed to Dr. Verne S. Caviness, Department of Neurology, Massachusetts General Hospital, 55 Fruit Street, VBK-901, Boston, MA 02114. E-mail: caviness@helix.mgh.harvard.edu. DOI:10.1523/JNEUROSCI.3091-07.2007

Copyright $\odot 2007$ Society for Neuroscience $\quad 0270-6474 / 07 / 2710777-08 \$ 15.00 / 0$ of the mapping system known to a PVE precursor? This fundamental question applies virtually to all proliferative epithelia that give rise to multiple cell classes in a temporal sequence (Melton, 1991; Jessell and Melton, 1992; Wolpert et al., 1998).

The classical model of positional mapping posits as an initiating state, the formation of a concentration gradient of a morphogen that diffuses from the margin of the field of specification induction. The morphogen concentration gradient then initiates differential expression of "master" transcription factors for different cell types. Whereas candidate soluble morphogens such as hedgehog and bone morphogenetic protein act inductively at the ventromedial and dorsolateral cerebral vesicle, their role in the TNG or transcriptional activation is not clear. It seems doubtful that an overall control mechanism based on morphogen diffusion could satisfy the where and when components of neocortical neurogenesis, especially in the massive extent of the PVE in primates and other gyrencephalic mammals.

In contrast, basic helix-loop-helix (bHLH) and homeobox transcription factors are expressed according to gradients aligned with the TNG (Nakagawa et al., 1999; Bulchand et al., 2001; O'Leary and Nakagawa, 2002; Grove and Fukuchi-Shimogori, 2003). These genes, singly or combinatorially (Reber et al., 2004) appear to be necessary to mechanisms of neuronal laminar class specification. However, how the where and when patterns of expression are regulated as the neuronal class specification advances during neurogenesis is not known.

We showed that coordinate regulation of two cell cycle parameters, probability of exit from the cycle (Q) and duration of 
the $G_{1}$ phase $\left(T_{G 1}\right)$, is intimately associated with mechanisms regulating successive generation of neocortical neuronal classes (Takahashi et al., 1995; Miyama et al., 1997; Caviness et al., 2000). However, whether $\mathrm{Q}$ or $\mathrm{T}_{\mathrm{G} 1}$ alone can specify the where and when components was not clear. Here, we incorporate a spatially graded parameter, namely, expression of the homeobox transcription factor Lhx2 (inferred from mRNA level) in the PVE, and show that, acting together, $\mathrm{Q}, \mathrm{T}_{\mathrm{G} 1}$, and Lhx2 could specify uniquely the where and when information. We suggest that this model can be generalized for linking positional information and cell class specification throughout the developing CNS.

\section{Materials and Methods}

Animals. Timed-pregnant FVB mice were obtained from Charles River Laboratories (Wilmington, MA) and maintained on a $12 \mathrm{~h}$ light/dark schedule in our animal facility. Pregnant dams carrying embryonic day 12 (E12), E13, or E14 mice (E0 is day of conception) were given injections of bromodeoxyuridine (BrdU; $50 \mu \mathrm{g} / \mathrm{g}$ body weight, i.p.; Sigma, St. Louis, MO). Injections were performed at 9:00 A.M. and repeated at 12:00 P.M. At 1:00 P.M. (i.e., $4 \mathrm{~h}$ after the first injection), the dams were anesthetized [ $50 \mu \mathrm{g} / \mathrm{g}$ Ketalar (Abbott Labs, Abbott Park, IL) and $10 \mu \mathrm{g} / \mathrm{g}$ Rompun (Bayer Biological Products, Clayton, NC)] and the embryos were removed by hysterotomy. All of the experimental procedures were in compliance with the institutional guidelines and the National Institutes of Health Guide for the Care and Use of Laboratory Animals.

Tissue processing and histology. Whole heads of the embryos were immersed in 4\% paraformaldehyde (PFA; Polysciences, Warrington, PA) in $0.1 \mathrm{M}$ PBS. After overnight immersion in the fixative, the heads were cryoprotected by immersion in $20 \%$ sucrose in $0.1 \mathrm{~m}$ PBS until they sunk (usually overnight). On the third day, brains were frozen on powdered dry ice and stored until further use at $-80^{\circ} \mathrm{C}$ in a freezer. The cryopreserved embryonic heads were sectioned on a cryostat (Frigocut 2800E; Leica, Bannockburn, IL) at a thickness of $12 \mu \mathrm{m}$ in the sagittal plane. Appropriate midsagittal sections were selected (with both the lateral as well as the medial ganglionic eminences and choroid plexus being visible) and processed alternately for BrdU immunohistochemistry or Lhx2 mRNA in situ hybridization.

$B r d U$ immunohistochemistry. The frozen sections were warmed to room temperature and incubated in $50 \%$ formamide in $2 \times$ SSC for $2 \mathrm{~h}$ at $65^{\circ} \mathrm{C}$. After rinsing two times in SSC for $5 \mathrm{~min}$, the sections were treated with $2 \mathrm{~N} \mathrm{HCl}$ for $30 \mathrm{~min}$ at $37^{\circ} \mathrm{C}$ and subsequently rinsed in $01 \mathrm{~m}$ boric acid, $\mathrm{pH}$ 8.5, for $5 \mathrm{~min}$. This was followed by another rinse, in $0.1 \mathrm{M}$ PBS for $5 \mathrm{~min}$, and incubation in $2 \% \mathrm{H}_{2} \mathrm{O}_{2}$ in PBS for $10 \mathrm{~min}$, to suppress endogenous peroxidase activity. The sections were rinsed three times in PBS for 5 min each. To block nonspecific antibody reaction, the sections were incubated in $3 \%$ normal horse serum with $0.1 \%$ Tween 20 in $0.1 \mathrm{M}$ PBS for $30 \mathrm{~min}$. Sections were incubated with mouse monoclonal antibody to $\operatorname{BrdU}$ (1:100; BD Biosciences, San Jose, CA), overnight at $4^{\circ} \mathrm{C}$. The next day, sections were rinsed in PBS. Then, biotinylated horse antimouse IgG (Vector Laboratories, Burlingame, CA) was applied for 60 $\mathrm{min}$ at room temperature. Sections were rinsed again, and the $\mathrm{ABC}$ peroxidase solution (ABC Peroxidase Elite kit; Vector Laboratories) was applied for another $60 \mathrm{~min}$ at room temperature. After a PBS rinse, the sections were reacted with diaminobenzidine with $\mathrm{H}_{2} \mathrm{O}_{2}$ (DAB Reagent Set; KPL, Gaithersburg, MD) for $4 \mathrm{~min}$. The sections were rinsed in distilled water, counterstained with $0.1 \%$ aqueous basic fuchsin, and coverslipped with Crystal Mount (Biomedia, Foster City, CA).

Analysis of cell cycle kinetic parameters. BrdU-labeled cells show a brown-colored nucleus, whereas unlabeled cells are red (basic fuchsin label). We counted BrdU-labeled and unlabeled cells in four sectors across the midsagittal axis, denoted as positions 1-4 (see Fig. 1C). Position 1 was at the rostral pole of the $\mathrm{VZ}$, and position 4 was at the caudal pole. Positions 2 and 3 were equidistant between positions 1 and 4 . Each sector was $100 \mu \mathrm{m}$ in width (i.e., its rostrocaudal extent) and $12 \mu \mathrm{m}$ in depth (i.e., the section thickness). Nuclei were counted with a $100 \times$ objective on a Zeiss (Thornwood, NY) microscope. The BrdU labeling index (LI) was calculated as the number of BrdU-labeled nuclei divided by the total number of nuclei per sector. Nonadjacent sections were used to avoid recounting the nuclei.

In each sector, the cell cycle duration $\left(\mathrm{T}_{\mathrm{C}}\right)$ and $\mathrm{T}_{\mathrm{G} 1}$ were calculated from the BrdU LI, using a formula (supplemental Fig. 1, available at www.jneurosci.org as supplemental material) based on the following considerations. The PVE is an asynchronously proliferating population of cells so that the fraction of cells in a given cell cycle phase at any moment corresponds to the ratio between the length of that phase and the length of the cell cycle. The growth fraction in this system is, for practical purposes, 1.0 (i.e., all cells in the PVE are proliferative, except for those postmitotic cells that are exiting). Therefore, if the PVE is continuously exposed to BrdU, the proliferating cells continue to become labeled as they pass through the $\mathrm{S}$ phase, and the LI increases linearly with a slope of $1 \div \mathrm{T}_{\mathrm{C}}$ until it reaches an asymptote corresponding to an LI of 1.0 at a time, $\mathrm{T}_{\mathrm{C}}-\mathrm{T}_{\mathrm{S}}$ (Nowakowski et al., 2002). A critical point, however, is that practically all cells in $\mathrm{S}$ phase label with BrdU within minutes of exposure, and thus the $y$-intercept is $\mathrm{T}_{\mathrm{S}} \div \mathrm{T}_{\mathrm{C}}$ [i.e., at the outset of the labeling experiment, the initial LI will correspond to the cells in $S$ phase as a fraction of the total cell population $\left(T_{S} \div T_{C}\right)$ ]. For our experiment, therefore, the LI will increase linearly as a function of the duration of exposure to BrdU. We used a cumulative labeling interval of $4 \mathrm{~h}$. Thus at $4 \mathrm{~h}$, the $\mathrm{LI}$ will correspond to $4 \div \mathrm{T}_{\mathrm{C}}+\mathrm{T}_{\mathrm{S}} \div \mathrm{T}_{\mathrm{C}}$. $\mathrm{T}_{\mathrm{S}}$ (duration of $S$ phase) is $\sim 4$ h (Takahashi et al., 1995, 1996; Miyama et al., 1997), so the equation reduces to $(4+4) \div \mathrm{T}_{\mathrm{C}}$ or $8 \div \mathrm{T}_{\mathrm{C}}$, so that we can calculate $\mathrm{T}_{\mathrm{C}}$ as $8 \div \mathrm{LI}$. To calculate $\mathrm{T}_{\mathrm{G} 1}$, we rearranged the basic equation $\mathrm{T}_{\mathrm{C}}=$ $\left(\mathrm{T}_{\mathrm{G} 1}+\mathrm{T}_{\mathrm{S}}+\mathrm{T}_{\mathrm{G} 2+\mathrm{M}}\right)$ to get $\mathrm{T}_{\mathrm{G} 1}=\mathrm{T}_{\mathrm{C}}-\left(\mathrm{T}_{\mathrm{S}}+\mathrm{T}_{\mathrm{G} 2+\mathrm{M}}\right)$. Because we know that $\mathrm{T}_{\mathrm{S}}$ is $\sim 4 \mathrm{~h}$ and $\mathrm{T}_{\mathrm{G} 2+\mathrm{M}}$ is $\sim 2 \mathrm{~h}$ (Takahashi et al., 1995, 1996; Miyama et al., 1997), we can calculate $T_{G 1}$ using the following equation: $T_{G 1}=T_{C}$ -6 .

$\mathrm{T}_{\mathrm{G} 1}$ data were converted from four positions to six positions by linear interpolation. The data for the anterior-most and posterior-most positions were unchanged by this manipulation. Each additional position in the six-position system was then derived from the four-position measured values by a linear weighting by distance. The fidelity of the conversion is shown in supplemental Figure 2 (available at www.jneurosci.org as supplemental material).

In situ hybridization of Lhx2 mRNA. In situ hybridization was performed by modifying a previously published protocol (Kerner et al., 1998). The sections were postfixed in $4 \%$ PFA, washed in $0.1 \mathrm{~m}$ PBS, and acetylated with acetic anhydride in tetraethylammonium, and the membranes were permeabilized in $1 \%$ Triton X-100 in $0.1 \mathrm{M}$ PBS, washed in $0.1 \mathrm{~m}$ PBS, and allowed to air dry under a fume hood. A radiolabeled antisense probe was generated by run-off transcription of the Lhx2 plasmid (kindly provided by Drs. D. D. M. O'Leary and Y. Nakagawa, The Salk Institute, La Jolla, CA) with SP6 RNA-polymerase in the presence of ${ }^{35} \mathrm{~S}$-CTP as label. To create the sense control probe, transcription was performed with T7 RNA-polymerase. The hybridization solution (containing formamide, $1 \mathrm{~m}$ Tris, $0.5 \mathrm{M}$ EDTA, $5 \mathrm{M} \mathrm{NaCl}, 50 \%$ dextran sulfate, and $50 \times$ Denhardt's solution, and in the probe mixture, salmon sperm, yeast total RNA, and yeast tRNA), as well as radioactive probe at a concentration of $172,500 \mathrm{dpm} / \mu$ l hybridization buffer, was applied and allowed to hybridize at $72^{\circ} \mathrm{C}$ overnight in a humid chamber. The slides were washed in $0.2 \times$ SSC, followed by $2 \times$ SSC, and then laid out to air dry. For quantification of the hybridization signal, we exposed the slides to autoradiographic film (Biomax MR; Eastman Kodak, Rochester, NY), including a radiographic $\mathrm{C}^{14}$ standard (Polysciences).

Quantitative analysis of Lhx2 mRNA expression. Relative optical densities (RODs) in the autoradiograms were measured using an MCID image analysis program (InterFocus Imaging, Linton, UK), and the data were stored in Excel (Microsoft, Redmond, WA) spreadsheets. ROD measurements were made in six equidistant sectors, located in the VZ, along the rostral-to-caudal axis in the midsagittal plane (see Fig. 3). The six sectors proved to sufficiently close to one another to give us confidence that the shape of the Lhx2 mRNA expression gradient could be detected with precision. At the same time, the six sectors were spaced sufficiently apart so as not to overlap with one another in any brain at any age group.

Laser capture microdissection and quantitative real-time PCR. Whole heads from E12-E14 embryos were frozen with isopentane cooled in liquid nitrogen. The heads were stored at $-80^{\circ} \mathrm{C}$ until further use. 
Twelve-micrometer-thick sections were cut in the sagittal plane on a cryostat, thaw mounted on noncoated glass slides (Gold-Seal RITE-ON micro slides; Gold Seal Products, Portsmouth, NH), and stored at $-80^{\circ} \mathrm{C}$. On the day of laser capture, the slides were allowed to equilibrate to room temperature, fixed in $70 \%$ ethanol, rehydrated, and counterstained with HistoGene (Arcturus, Mountain View, CA). The sections were dehydrated in ethanol and cleared in xylene for $2 \mathrm{~min}$.

The VZ was excised and captured using the PixCell-II LCM instrument (Arcturus). The $\mathrm{VZ}$ was identified for laser capture based on previous BrdU-labeling data (Takahashi et al., 1995), cell packing, and cell orientation (revealed by the counterstain). From each age, four equalsized sectors of the VZ, equidistantly spaced along the midsagittal axis, were captured. The captured tissue was solubilized in extraction buffer (Pico Pure RNA isolation kit; Arcturus) for $30 \mathrm{~min}$ at $42^{\circ} \mathrm{C}$ and stored at $-80^{\circ} \mathrm{C}$.

RNA was extracted using the same kit, and reverse transcription was performed using the Superscript II First-Strand Synthesis System for reverse transcription-PCR (Invitrogen, Carlsbad, CA) using random hexamer primers according to the manufacturer's instructions.

Quantitative real-time PCR was performed with a Bio-Rad (Hercules, CA) iCycler by using the SYBR-Green PCR Master Mix (Applied Biosystems, Foster City, CA) through 50 PCR cycles $\left(95^{\circ} \mathrm{C}\right.$ for $30 \mathrm{~s}, 57^{\circ} \mathrm{C}$ for $60 \mathrm{~s}, 72^{\circ} \mathrm{C}$ for $90 \mathrm{~s}$ ). Each cDNA sample was run in triplicate for the target and the endogenous control gene [glyceraldehyde-3-phosphate dehydrogenase (GAPDH)] in the same 96-well plate. Specificity of amplicons was determined by melt-curve analysis and DNA sequencing. Primer pair sequences were designed using Primer3 (http://frodo.wi.mit.edu/) and were as follows: Lhx2 (GenBank accession number NM_010710.2), CCAGCTTCGGACAATGAAGT (forward) and TTTCCTGCCGTAAAAGGTTG (reverse); GAPDH, ATGACATCAAGAAGGTGGTG (forward) and CATACCAGGAAATGAGCTTG (reverse).

Statistical analyses. For each type of experiment, we collected data from five brains for each age group, and from four, four, and five litters and 12, 14, and 24 sections for E12, E13, and E14, respectively. Statistical analysis was performed using Microsoft Excel. ANOVA and Student's $t$ tests were performed with $p<0.05$ denoting statistical significance.

\section{Results}

\section{Lhx2 mRNA expression gradients}

Lhx2 mRNA expression was the greatest at the posterior pole (position 6) and lowest at the anterior pole at its border with the striatum (position 1), consistent with previous reports (O'Leary and Nakagawa, 2002). The Lhx2 signal intensity increased as an orderly gradient in parallel with the sagittal axis. Qualitatively, the changes in the gradient are gradual and apparently continuous and uniform across the surface of the PVE (Fig. $1 B$ ) (i.e., there were no apparent sharp step changes suggestive of "border" transitions). This qualitative impression was confirmed with densitometric measurements (Fig. 2).

A two-dimensional plot of Lhx2 mRNA levels versus position along the sagittal plane for each age (Fig. $2 \mathrm{~A}$ ) shows an anterior to posterior increase in expression level at each age. Statistical analysis (ANOVA), however, shows that expression at the anteriormost position does not change significantly during the E12-E15 period. Therefore, we "anchored" the anterior pole at the nominal value of 1.0, which we assigned to the youngest age, E12, to permit comparison of the slopes of the gradients of expression across the E12-E15 interval. Whereas levels of Lhx2 mRNA expression are the same for the anterior-most positions across age, the levels at the successively posterior positions decline with age. That is, the slope of the expression gradient declines such that, for any given position, the highest levels are measured at E12 and the levels progressively decline toward E15. This is illustrated in a three-dimensional surface plot (Fig. $2 \mathrm{~B}$ ). The rising levels of Lhx2 expression from anterior to posterior positions at each age
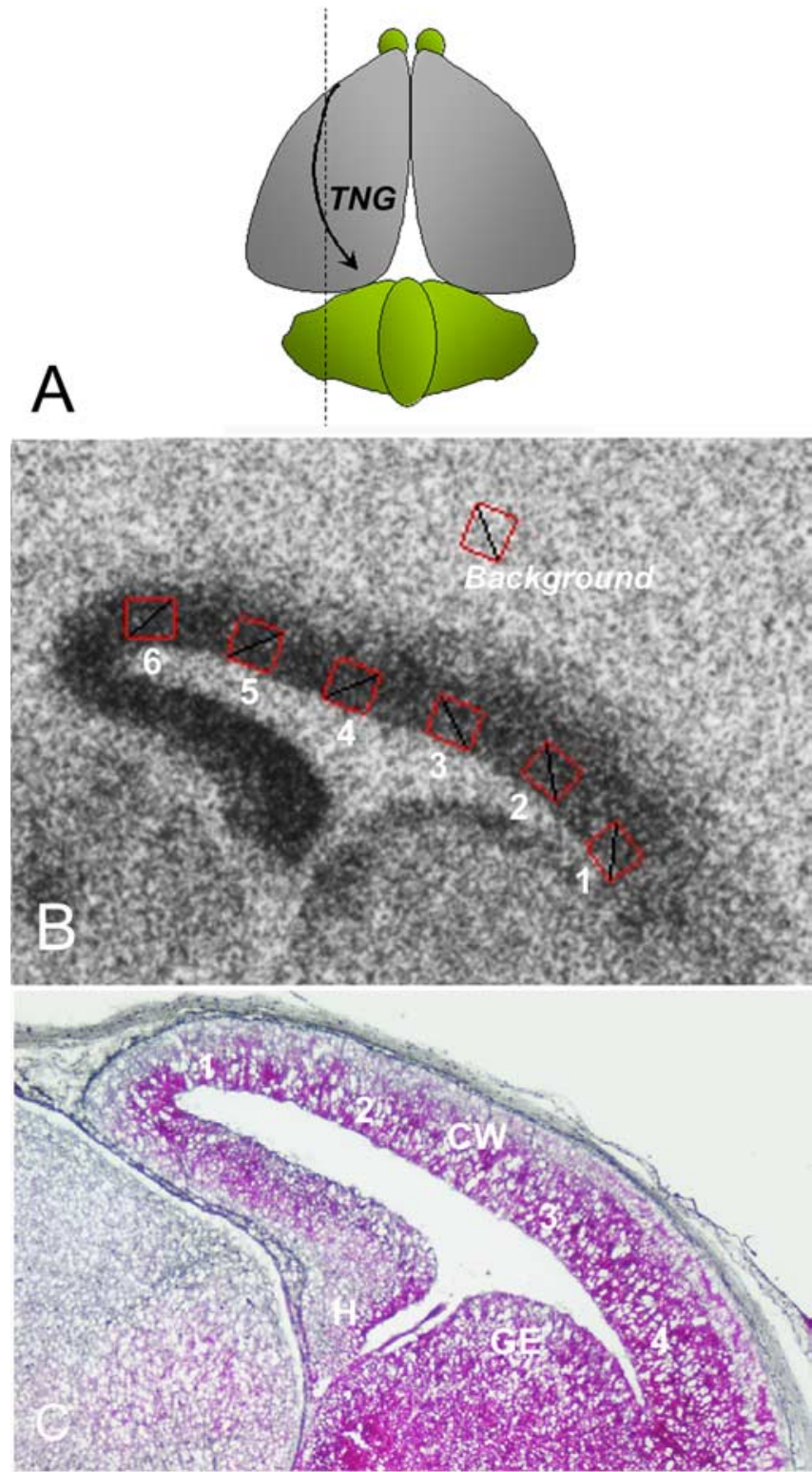

Figure 1. $A$, A schematic diagram showing the approximate orientation and direction of the TNG (black arrow). $\boldsymbol{B}$, Midsagittal section of E14 mouse cerebral wall showing in situ hybridization for Lhx2 mRNA. Quantification of the mRNA expression was performed in the VZ only at six equidistant positions, as indicated by the red boxes. Intensity of the background signal was quantified in a box off of the sections. $\boldsymbol{C}$, BrdU labeling indices were calculated at four equidistant anterior to posterior positions (1-4) in midsagittal sections through the E14 mouse cerebral wall. CW, Cerebral wall; GE, ganglionic eminence; $H$, hippocampal formation.

potentially allow the Lhx2 level to serve as a positional code. However, the decline in Lhx2 expression at the posterior pole together with the fixed level at the anterior positions across time means that Lhx 2 expression alone is insufficient to specify position on the gradient across ages. That is, there is no unique association of specific expression levels of Lhx2 with either a specific time or specific location in the PVE across the E12-E15 interval. For example, the value of 1.5 units of Lhx 2 expression is present at all possible cortical positions across the E12-E15 interval (i.e., quite anterior at E12 and E13 but quite posterior at E15). Thus, it is clear that a PVE cell cannot use an Lhx2 expression level "readout" alone to compute its anteroposterior position at all times through the neurogenetic interval. 


\section{A. Lhx2 mRNA Expression Gradients}

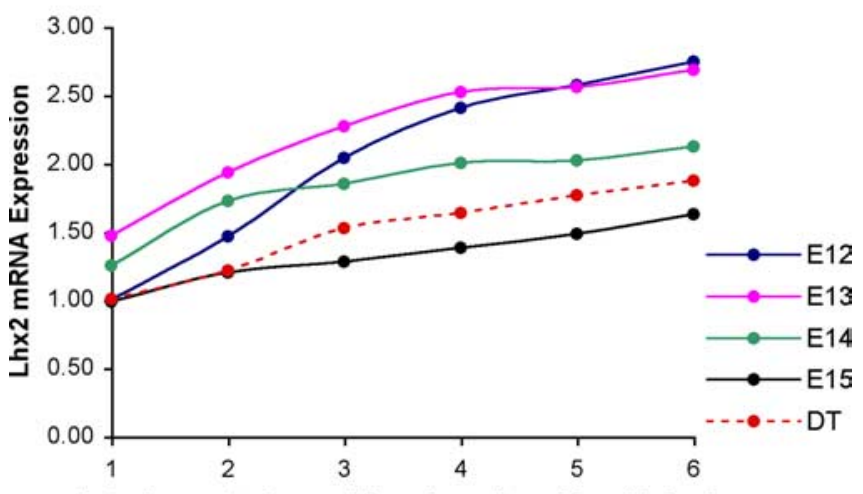

Anterior-posterior position along the midsagittal axis

\section{B. Surface Plot of Lhx2 mRNA expression}

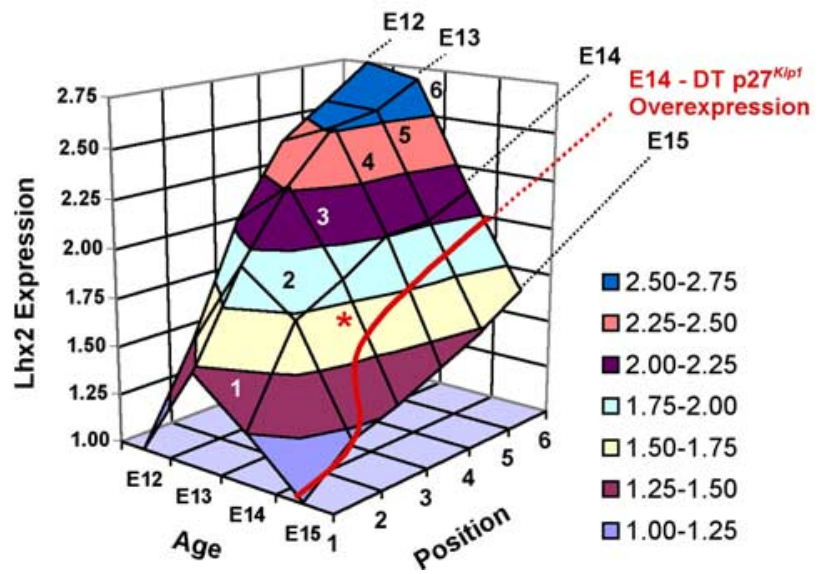

Figure 2. Gradients of Lhx2 expression. The gradients of Lhx2 expression as aligned along the midsagittal plane plotted against embryonic age in WT and DT embryos. Positions from anterior to posterior along the midsagittal plane are numbered from 1 (anterior pole) to 6 (posterior pole). Embryonic age spans the interval E12-E15.A, The slope of Lhx2 mRNA expression ascends from anterior (position 1) to posterior (position 6) at each age from E12 to E15 in WT mice. However, the slopes decrease during the interval E12-E15. Data from each age in WT mouse are shown with solid lines, and the data from the E14 DT mouse (with p27 ${ }^{\text {Kip } 1}$ overexpression) are shown with a dashed line. The levels are approximately constant at position 1 throughout the E12-E15 period. $\boldsymbol{B}, A$ three-dimensional surface plot of the data in $\boldsymbol{A}$ to show the interrelationship of Lhx2 levels, age, and anterior to posterior position along the midsagittal plane. The relative levels of $L h \times 2$ expression determined by densitometry are indicated on the vertical axis, the age of the embryo is indicated on the horizontal axis to the left, and the anterior to posterior position is indicated on the horizontal axis to the right. The levels of Lhx2 expression are approximately constant at the most anterior position (position 1). Lhx2 expression at position 1 at each age is assigned a nominal value of 1 , and expression along the midsagittal plane at each age is normalized with respect to this value at position 1. The age (E12-E15) isobars trace from the age axis (left horizontal) in parallel with the position axis (right horizontal). The thick solid red line traces the gradient of Lhx2 expression in the E14 DT mouse (equivalent to the dashed line in $A$ ), which is shifted away from the E14 and toward the E15 WT expression. The offset in Lhx2 expression levels between the WT and DT E14 isobars along the position 3 isobar (asterisk) represents the Lhx2 expression-level error induced by p27 ${ }^{\text {Kip } 1}$ overexpression at position 2 (see also Table 2).

Confirmation of the range of Lhx $2 \mathrm{mRNA}$ expression by laser capture microdissection and quantitative real-time PCR

The in situ hybridization technique used here indicates that the relative levels of Lhx 2 expression fall within the same range over the entire E12-E14 interval. This was confirmed by quantitative real-time PCR in samples of E12, E13, and E14 VZ obtained by laser capture microdissection (supplemental Fig. 3, available at www.jneurosci.org as supplemental material). The absolute values of Lhx 2 mRNA levels obtained by the two methods cannot, of course, be equated in that the absolute levels cannot be determined by in situ hybridization.

\section{The cell cycle progression gradient}

The BrdU LI after $4 \mathrm{~h}$ of cumulative BrdU labeling was determined at four equidistant positions along the $\mathrm{VZ}$ in the midsagittal plane (Fig. 1C). The LI at each position for each age along with the corresponding $\mathrm{T}_{\mathrm{G} 1}$ and $\mathrm{T}_{\mathrm{C}}$ are given in Table 1. Because the Lhx2 data were analyzed at six equidistant positions, for convenience of comparing $\mathrm{T}_{\mathrm{G} 1}$ with Lhx2 mRNA, we converted the four-position $\mathrm{T}_{\mathrm{G} 1}$ data to six-position data using linear interpolation (Table 2) (see Materials and Methods and supplemental Fig. 2, available at www.jneurosci.org as supplemental material). $\mathrm{T}_{\mathrm{G} 1}$ increases from $\sim 4 \mathrm{~h}$ posteriorly at E12 to nearly $11 \mathrm{~h}$ anteriorly at E14 (Fig. 3A). In contrast to the Lhx2 gradient, the changes in $\mathrm{T}_{\mathrm{G} 1}$ are non-overlapping across age such that all points at $\mathrm{E} 13$ have a longer $\mathrm{T}_{\mathrm{G} 1}$ than all points at E12, and similarly all points at E14 have a longer $\mathrm{T}_{\mathrm{G} 1}$ than all points at E13. As with the Lhx2 gradients, visual inspection of the sections at each age shows that the changes in LI appear to be continuous (i.e., there are no obvious step changes in the slopes at any point along the anteriorto-posterior axis). The continuity of LI descent with corresponding increase in $\mathrm{T}_{\mathrm{G} 1}$ from posterior to anterior at each age and across the successive ages is seen more clearly in the threedimensional surface plot shown in Figure $3 B$. These changes in $\mathrm{T}_{\mathrm{G1}}$ are in accordance with previous observations obtained in the coronal plane (Takahashi et al., 1995; Miyama et al., 1997).

The value of $\mathrm{T}_{\mathrm{Gl}}$, as it increases with time at each point of the TNG, is correlated with the proportion of the neurogenetic interval that has elapsed at that point (Takahashi et al., 1995; Miyama et al., 1997). It is also correlated with the specific laminar classes of cells that are arising at that point in the PVE (Takahashi et al., 1999). Thus, the rise of $T_{G 1}$ can serve as a "clock" for the PVE cells to time advance through the succession of cell classes arising. Because the Lhx2 gradient varies systematically with time, the expression level of Lhx2 at any point on the PVE will be specific to a time point in the neurogenetic interval. That is, for any point in the PVE and at any time in the neurogenetic interval, the value of $\mathrm{T}_{\mathrm{G} 1}$ informs a cell the when information with respect to laminar neuron class sequence, whereas Lhx2 level informs the cell the where information with respect to position in the PVE. The two parameters in combination become a unique descriptor for both the when and where information needed by a PVE cell (Fig. $4 A$ ).

The increase in $\mathrm{T}_{\mathrm{G} 1}$ approaches asymptote at E14. It would mean that the when information becomes less specific at E15, and thus the combinatorial unique descriptor is most useful only from E12 to E14. This is to be expected and does not lessen the power of the model in that, by late E14, the final laminar cell classes to be formed (i.e., those of the superficial layers II and III) are already arising from the PVE in all neocortical areas (Takahashi et al., 1999). Thus, by the time $\mathrm{T}_{\mathrm{G} 1}$ approaches its asymptote, all neuronal classes have already been specified and additional when information is no longer needed.

\section{Lhx2 expression in a double-transgenic mouse model of} p27 ${ }^{\text {Kip1 }}$ overexpression in the PVE

We have shown previously that the normal mouse $\mathrm{T}_{\mathrm{G} 1}$ and another cell cycle parameter, Q (the cell cycle output fraction), are coordinately regulated at each of the 11 cell cycles throughout the 7 d neurogenetic interval (Takahashi et al., 1995; Miyama et al., 1997). We also have shown that when $\mathrm{p} 27^{\mathrm{Kip} 1}$ is overexpressed in 
Table 1. BrdU LI and $\mathrm{T}_{\mathrm{G} 1}$

\begin{tabular}{|c|c|c|c|c|c|c|c|}
\hline Position & & & E12 & E13 & E14 & E15 & E14 DT \\
\hline$A^{a}$ & 1 & LI & $0.68 \pm 0.02$ & $0.59 \pm 0.01$ & $0.47 \pm 0.01$ & $0.48 \pm 0.01$ & $0.54 \pm 0.01$ \\
\hline & & $T_{61}$ & $5.72 \pm 0.16$ & $7.67 \pm 0.18$ & $11.09 \pm 0.29$ & $10.77 \pm 0.27$ & $8.71 \pm 0.29$ \\
\hline & 2 & LI & $0.74 \pm 0.02$ & $0.62 \pm 0.01$ & $0.49 \pm 0.01$ & $0.49 \pm 0.01$ & $0.55 \pm 0.01$ \\
\hline & & $\mathrm{T}_{\mathrm{G} 1}$ & $4.80 \pm 0.10$ & $6.89 \pm 0.26$ & $10.29 \pm 0.23$ & $10.38 \pm 0.38$ & $8.42 \pm 0.23$ \\
\hline & 3 & $\mathrm{LI}$ & $0.76 \pm 0.02$ & $0.65 \pm 0.02$ & $0.52 \pm 0.01$ & $0.50 \pm 0.02$ & $0.59 \pm 0.01$ \\
\hline & & $\mathrm{T}_{\mathrm{G} 1}$ & $4.52 \pm 0.09$ & $6.34 \pm 0.24$ & $9.44 \pm 0.26$ & $10.15 \pm 0.36$ & $7.57 \pm 0.26$ \\
\hline & 4 & LI & $0.76 \pm 0.02$ & $0.66 \pm 0.02$ & $0.53 \pm 0.02$ & $0.54 \pm 0.03$ & $0.62 \pm 0.02$ \\
\hline & & $\mathrm{T}_{\mathrm{G} 1}$ & $4.52 \pm 0.10$ & $6.08 \pm 0.21$ & $9.09 \pm 0.32$ & $8.77 \pm 0.32$ & $6.82 \pm 0.32$ \\
\hline $\mathrm{B}^{b}$ & 1 & & 5.47 & 7.54 & 11.01 & 10.95 & 8.86 \\
\hline & 2 & & 5.24 & 7.22 & 10.60 & 10.58 & 8.47 \\
\hline & 3 & & 5.01 & 6.91 & 10.19 & 10.21 & 8.07 \\
\hline & 4 & & 4.77 & 6.59 & 9.77 & 9.83 & 7.68 \\
\hline & 5 & & 4.54 & 6.27 & 9.36 & 9.46 & 7.29 \\
\hline & 6 & & 4.31 & 5.95 & 8.95 & 9.09 & 6.90 \\
\hline
\end{tabular}

${ }^{a}$ Mean \pm SEM values for BrdU LI and $T_{G 1}$ (in hours) for each of the four anterior-to-posterior positions analyzed. The $L I$ is the ratio between BrdU-labeled cells and total cells in the $V Z$ in midsagittal sections after a $4 \mathrm{~h}$ BrdU exposure.

${ }^{b}$ The $\mathrm{T}_{\mathrm{G} 1}$ data obtained from analyses at four equidistant positions were converted by linear interpolation (see Materials and Methods and supplementa Fig. 2, available at www.jneurosci.org as supplemental material) to six-point data (shown for B) for optimal comparison with the Lhx2 data (Figs. 3, 4). Position 1 is at the anterior pole, and position 6 is at the posterior pole.

Table 2. Correlation of Lhx2 mRNA expression with cell cycle parameters $T_{G 1}, \mathbf{Q}$, and laminar class of neurons arising over the interval E12-E14 in WT mice and on E14 in a DT mouse that overexpresses the cell cycle inhibitor p27 $27^{\text {Kip } 1}$

\begin{tabular}{lllll}
\hline & E12 & E13 & E14 & E14DT \\
\hline Lhx2 mRNA & 2.25 & 2.23 & 1.85 & 1.35 \\
$\mathrm{~T}_{\mathrm{G} 1}(\mathrm{~h})$ & $4.52 \pm 0.09$ & $6.34 \pm 0.24$ & $9.44 \pm 0.26$ & $7.57 \pm 0.26$ \\
$\mathrm{Q}$ & $\mathrm{NA}$ & $\mathrm{NA}$ & 0.35 & 0.40 \\
$\%$ SG & $\mathrm{NA}$ & $\mathrm{NA}$ & $21.5 \pm 8.1$ & $41.7 \pm 7.0$ \\
\hline
\end{tabular}

The values for $\mathrm{T}_{\mathrm{G} 1}$ and $\mathrm{Lh} \times 2 \mathrm{mRNA}$ expression are from position 3 along the anteroposterior axis in the present analysis, whereas the values for the other parameters are from a comparable location published in a previous study (Tarui et al., 2005). Supragranular layers (SG) refer to the aggregate of layers IV, III, and II. \%SG refers to the percentage of all neocortical cells that are destined to the SG layers. In the E14 DT embryo, Lhx2 mRNA expression decreases, the value of $Q$ increases, and a larger percentage of neurons are destined for $S G$ layers compared with the E14 WT embryo. Thus, in the DT mouse, neuronal laminar class covaries directly with $Q$ and inversely with Lhx2 mRNA levels, whereas $\mathrm{T}_{\mathrm{G} 1}$ is independent of these parameters.

the PVE in the double-transgenic (DT) mouse, the normal tight correlation between $\mathrm{T}_{\mathrm{G} 1}$ and $\mathrm{Q}$ is dissociated (Mitsuhashi et al., 2001; Tarui et al., 2005). That is, Q is increased without any change in $\mathrm{T}_{\mathrm{G} 1}$. Moreover, in this DT mouse, the sequence of laminar neuronal class origin is also perturbed so that production of neurons of the superficial laminas II-III begins prematurely during the 11-cell cycle and $7 \mathrm{~d}$ neurogenetic period. However, whether Lhx2 expression, which is the where component in our positional encoding model, also changed in the DT mouse was not known.

We found that although the Lhx 2 mRNA expression exhibited an orderly graded ascent from the anterior-most to the posteriormost positions of the TNG in the DT embryos and although the gradient was anchored at 1.0 at the anterior pole, Lhx2 mRNA levels at successively posterior positions were intermediate between values at E14 and E15 rather than being at E14 values obtained for the WT animal (Fig. 2). Thus, the Lhx2 gradient had advanced prematurely in the DT embryos (Fig. 2), in line with the premature advance of Q (Mitsuhashi et al., 2001; Tarui et al., 2005).

We measured BrdU LI in the DT embryos using the same methods as in the WT embryos and calculated $\mathrm{T}_{\mathrm{G} 1}$ (Table 1 and supplemental Fig. 1, available at www.jneurosci.org as supplemental material). The BrdU LI in the E14 DT animals corresponded approximately to that expected for E13.5 in WT embryos. Although the DT embryos were nominally E14, because of variability among and within litters, the average developmental age of the DT mice is assumed to be E13.5. As noted above, the expression levels of Lhx2 in the DT embryos corresponded approximately to those that would occur at E14.5 in WT embryos (Figs. 2, 3). The BrdU LI and Lhx2 measurements were made in the same embryos, so these shifts clearly represent a $\mathrm{p} 27^{\text {Kip } 1}$-induced separation of the control of $\mathrm{T}_{\mathrm{G} 1}$ versus Lhx2. Because $\mathrm{T}_{\mathrm{G} 1}$ has been previously established not to be altered by p $27^{\text {Kip } 1}$ overexpression (Tarui et al., 2005), it is apparent that $\mathrm{p} 27^{\text {Kip } 1}$ overexpression does, in contrast, induce an advance in the levels of Lhx2 mRNA relative to $\mathrm{T}_{\mathrm{Gl}}$. Similarly, because $\mathrm{Q}$ has been shown previously to advance by the p $27^{\text {Kip } 1}$ overexpression (Tarui et al., 2005), Lhx2 and Q may be regulated by interdependent mechanisms. In summary, as illustrated in Table 2, these experiments demonstrate that Lhx2 mRNA expression, $\mathrm{Q}$, and laminar cell class origin are correlated with each other while being dissociated from $\mathrm{T}_{\mathrm{Gl}}$.

\section{Discussion}

The neocortical PVE gives rise to an invariant sequence of laminar neuron classes and according to the precisely ordered TNG. This implies that, at all times in the neurogenetic interval, a PVE cell knows where it is with respect to the TNG and when it is with respect to laminar neuron class succession. We identify two candidate coordinate systems that interactively are sufficient to specify a unique place and time in the PVE. Our candidate for the where mechanism is Lhx2, a LIM homeobox transcription factor, and our candidate for when is $\mathrm{T}_{\mathrm{G} 1}$ a cell cycle kinetic parameter. Importantly, neither candidate alone is sufficient to specify a unique place and time.

Lhx2 is among other homeobox and bHLH transcription factors that are expressed in the PVE according to gradients aligned with the TNG and that are implicated in cell class specification (Porter et al., 1997; Bulchand et al., 2001; Monuki et al., 2001; Nakagawa and O'Leary, 2001; O'Leary and Nakagawa, 2002; Schuurmans and Guillemot, 2002). Lhx2 is expressed in a low anterior and high posterior gradient (i.e., in a direction opposite to that of the TNG). We regard Lhx2 as a proxy for the transcription factors collectively expressed as gradients aligned with the TNG and implicated in mechanisms of specification (Reber et al., 2004).

Why does Lhx2 fulfill the criteria for the where component of the positional encoding system? The key observation in support of this is that the expression level of Lhx2 at the anterior pole of the neocortical PVE does not change significantly at any time during E12-E15, but the levels change posteriorly during the same interval. However, Lhx 2 expression cannot uniquely specify the position of a proliferating cell lineage in the TNG because, depending on time, (1) the level of its expression at any given location is variable and (2) a given expression level over a wide range is encountered over most of the extent of the TNG. Therefore, another source of information is needed for the when component with respect to the laminar class specification sequence.

Our candidate for the when mechanism is $\mathrm{T}_{\mathrm{G} 1}$ because a specific range of $\mathrm{T}_{\mathrm{Gl}}$ is associated with the production of specific laminar neuronal classes throughout the PVE (Takahashi et al., 


\section{A. $T_{\mathrm{G} 1}$ Gradients}

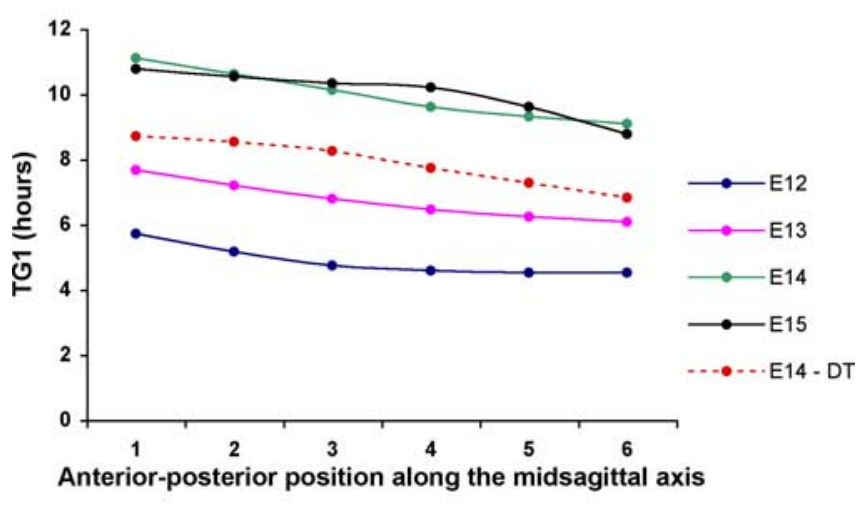

\section{B. Surface Plot of $T_{G 1}$}

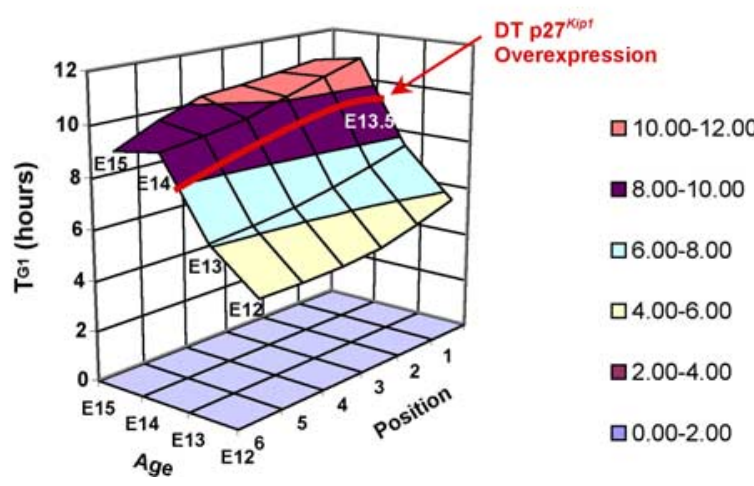

Figure 3. Gradients of duration of the $G_{1}$ phase. The gradients of $T_{G 1}$ derived from BrdU labeling indices as aligned along the midsagittal plane are plotted against embryonic age. Positions from anterior to posterior along the midsagittal plane are numbered from 1 (anterior pole) to 6 (posterior pole). Embryonic age spans the interval E12-E15. $A, T_{G 1}$ increases from anterior (position 1) to posterior (position 6) (i.e., with slope opposite in direction with reference to that of the Lhx2 mRNA expression gradient) (see Fig. 2). $\mathrm{T}_{\mathrm{G} 1}$ is shortest at E12 and longest at $\mathrm{E} 15$ and increases with age without overlap at successive ages. Data from each age in WT are shown with a solid line; the dashed line shows the data from the DT embryos at E14. The age-related increases in $\mathrm{T}_{\mathrm{G} 1}$ are approximately uniform throughout the anterior-to-posterior extent at each age, and there is an approximately continuous increase from age to age. $\boldsymbol{B}$, The data in $\boldsymbol{A}$ are plotted as a three-dimensional surface plot to show the interrelationship of $\mathrm{T}_{61}$, age, and anterior to posterior position along the midsagittal plane. (Note that the orientation of axes for position and embryonic age are the reverse of that in Fig. $2 B$ to facilitate illustration of the changes along the surface optimally.) The horizontal strips show that ranges of expression of $\mathrm{T}_{\mathrm{G} 1}$ increase fairly uniformly as a function of age across the entire anterior-to-posterior extent of the hemisphere but approach an asymptote in the E14-E15 interval. The thick solid red line shows that the gradient of $\mathrm{T}_{G 1}$ for the DT mouse at E14 (the dashed line from $A$ ) is shifted toward an earlier, approximately E13.5, pattern.

1999). Thus, a unique value of $\mathrm{T}_{\mathrm{G} 1}$ correlates with a unique laminar neuron class. However, $\mathrm{T}_{\mathrm{G} 1}$ alone cannot inform a lineage as to where it is in the TNG because each lineage in all locations along the TNG progresses through the full range of $\mathrm{T}_{\mathrm{G} 1}$, so that $\mathrm{T}_{\mathrm{G} 1}$ has no positional specificity. However, if a PVE cell were to combine its values of $\mathrm{T}_{\mathrm{G} 1}$ and that of $\mathrm{Lhx} 2$ expression, it would be informed simultaneously about both its where and when status (Fig. 4).

This combination of two information sources as a solution to determining a unique position on a surface is not unlike that achieved in the 18th century by the invention of the nautical clock (Sobel, 1995). This was a necessary technological advance because astronomical readings are sufficient to determine position at sea only if the time of day is accurately known. For our navigational hypothesis, astronomical observations are analogous to Lhx2 expression levels, whereas an accurate time would be anal-

\section{A. $T_{G 1}$ versus Lhx2 mRNA in wild type embryo}

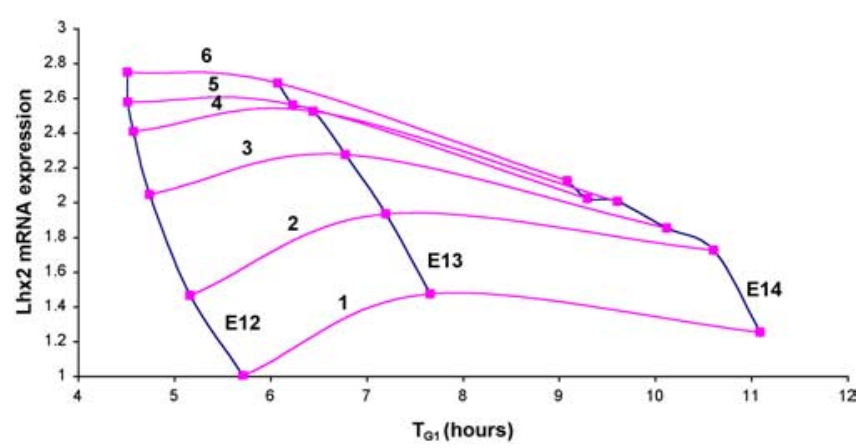

B. $T_{\mathrm{G} 1}$ versus Lhx2 mRNA in DT embryo showing shift in Lhx2

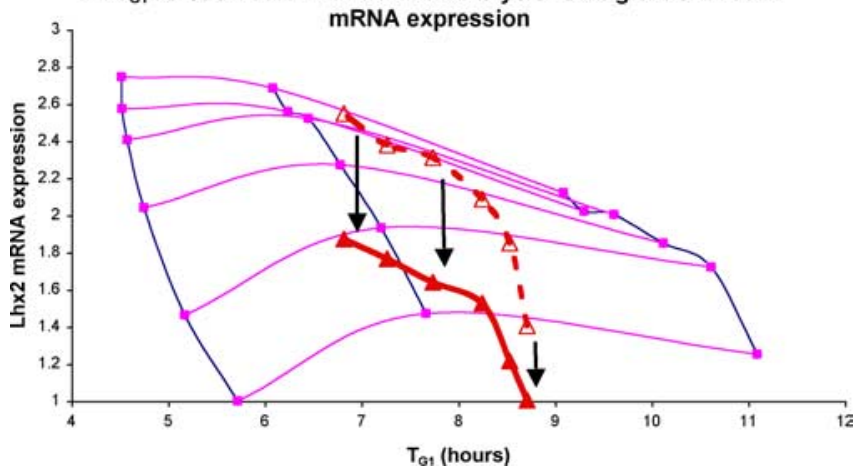

Figure 4. Intersections of values of $\mathrm{T}_{\mathrm{G} 1}$ and $\mathrm{Lh} \times 2 \mathrm{mRNA}$ at each anteroposterior position (1-6) at E12-E14. $\boldsymbol{A}$, Intersections in wild type. The blue lines (approximately vertical and ordered from left to right) interconnect the data from the same age (i.e., E12-E14). The magenta lines (approximately horizontal and ordered vertically from anterior to posterior) interconnect the data from each of the six anterior to posterior positions. This plot shows that the intersection of each pair of $L h \times 2$ and $\mathrm{T}_{G 1}$ data points defines a unique "when-where" condition in the TNG. $\boldsymbol{B}$, Intersections in $\mathrm{p} 27^{\text {Kip }}$ overexpression DT on E14 in relation to the WT grid. The solid red line shows the Lhx2 and $T_{G 1}$ data from the DT animal; the red dashed line shows hypothetical Lhx2 and $T_{G 1}$ values from the DT animal if the Lhx2 expression level had corresponded to the $\mathrm{T}_{G 1}$ data in wild type. Corresponding points of intersection on the $\mathrm{T}_{G 1}$ axis for the observed and WT-predicted Lhx2 values in DT animals are registered by vertical arrows. The downward shift from the red dashed line to the red solid line represented by the arrows shows, therefore, the "error" in the DT animal as a shift toward a more anterior TNG identity, or where condition, at any given $\mathrm{T}_{\mathrm{G}}$.

ogous to $\mathrm{T}_{\mathrm{G} 1 \text {. }}$ Thus, the combined cues of $\operatorname{Lhx} 2$ and $\mathrm{T}_{\mathrm{G} 1}$, as a "navigational hypothesis," are integrated to specify precisely the where in the TNG and the when in the laminar neuron progeny fate sequence for a given PVE cell (Fig. 4). We recognize that both time and astronomical coordinates are external to the seafaring vessel, whereas both Lhx2 and $\mathrm{T}_{\mathrm{G} 1}$ are intrinsic to the PVE cells. However, this difference does not detract from our analogy in that, in our model, a PVE cell can "compute" its location in the TNG at any time during the neocortical neurogenetic sequence by integrating information from two independent sources namely, $\mathrm{Lhx} 2$ and $\mathrm{T}_{\mathrm{G} 1}$.

$\mathrm{T}_{\mathrm{G} 1}$ values are correlated with the cell output parameter $\mathrm{Q}$ in each of the 11 cell cycles over the $7 \mathrm{~d}$ neurogenetic period in the WT mouse (Takahashi et al., 1995, 1996; Miyama et al., 1997). Thus, specific ranges of not only $\mathrm{T}_{\mathrm{G} 1}$ but also $\mathrm{Q}$ are associated with specific laminar neuronal classes (Takahashi et al., 1999). What is the role of $Q$ in our model of positional encoding system? Is it redundant because it overlaps with $\mathrm{T}_{\mathrm{G} 1}$ ? Our $\mathrm{p} 27^{\text {Kipl }}$ overexpression DT mouse offers unique insights into this issue. In the DT mouse, Lhx2 expression levels, values of $\mathrm{Q}$, and also the profile of neuronal classes arising are shifted toward a more mature 
or anterior position along the TNG. That is, production of superficial (layers II-III) laminar neuron classes begins earlier than normal (Table 2) (Tarui et al., 2005). This finding links Q, cell classes arising, and Lhx2 expression levels. Importantly, this linkage occurs with a simultaneous dissociation between the physiological tight linkage between $\mathrm{T}_{\mathrm{G} 1}$ and $\mathrm{Q}$ (Tarui et al., 2005). Two significant insights emerge from this observation. First, independent mechanisms regulate $\mathrm{T}_{\mathrm{G} 1}$ and Lhx2 expression, critical evidence in support of the role of these two parameters in our positional encoding system. Second, when Q is prematurely advanced, it advances Lhx2 expression to a more "mature" position along the TNG because of the tight linkage between Q and Lhx2. That is, the $\mathrm{p} 27^{\text {Kip } 1}$ overexpression-induced values of $\mathrm{Q}$ and $\mathrm{Lhx} 2$ at a given value of $\mathrm{T}_{\mathrm{Gl}}$ in $\mathrm{DT}$ animals are appropriate to higher values of $\mathrm{T}_{\mathrm{G} 1}$ with reference to $\mathrm{Q}$ and Lhx2 expression levels in the wild type. This perturbation of the where component leads the PVE cells astray in the navigational chart as the PVE cell "misreads" its $\mathrm{T}_{\mathrm{G} 1}$ value to "match" the new where information provided by the altered Lhx2 levels and begins producing neuronal classes appropriate for the new, albeit mismatched, position along the TNG (laminar classes II-III) (Fig. 4A, Table 2).

Other lines of evidence show that alteration of $\mathrm{Q}$ leads to improper laminar class production, and we have previously proposed a formal model for this mechanism of supragranular layer impoverishment (Caviness et al., 2003). For example, mutationinduced increase in the rate of advance of Q leads ultimately to impoverishment of supragranular neuronal populations in multiple mouse models. Specific examples of genes that show this phenotype include Id (Yun et al., 2004), Tlx (Roy et al., 2004), and Lhx2 (Monuki et al., 2001). Such laminar disorders, plausibly with the same underlying mechanism, are also characteristic of the human microcephaly vera malformation (Hammarberg, 1895; Cunningham, 1985; Caviness et al., 2003; Caviness et al., 2007). In these scenarios, alteration of $Q$ may lead to perturbation of the where component resulting in a misreading of the when component in our navigational model.

That $\mathrm{p} 27^{\text {Kip } 1}$, Lhx2, and $\mathrm{Q}$ are interdependent, and $\mathrm{T}_{\mathrm{G} 1}$ is independently regulated, is not unexpected. Variations in $T_{G 1}$ arise from alterations in early postmitosis, prerestriction point mechanisms directed at phosphorylation of $\mathrm{Rb}$ (Zetterberg et al., 1995; Novak and Tyson, 2003, 2004). Q, in contrast, is influenced by the antagonistic relationship between notch signaling and p27 ${ }^{\text {Kipl }}$ (Murata et al., 2005; Sarmento et al., 2005). The transcription factor Hes1, driven by the notch intracellular domain (NICD), is a critical player in this balance. Thus, a heteromer of NICD, binding with centromere binding factor, activates Hes1, which in turn suppresses the neuronal specification transcription cascades (Yoon and Gaiano, 2005). Moreover, p27 ${ }^{\text {Kip } 1}$ transcription is also suppressed by Hes1 and by Hes1-independent and proteasome-dependent mechanisms (Murata et al., 2005; Sarmento et al., 2005).

Thus, we propose that the antagonistic relationship between notch and $\mathrm{p} 27^{\text {Kip } 1}$ links $\mathrm{p} 27^{\text {Kip } 1}$, Lhx2, and neuronal class specification. That is, mechanisms that increase $\mathrm{p} 27^{\mathrm{Kip} 1}$ not only increase Q but also, through NICD downregulation, decrease the suppressive effect of Hes 1 during advance in the cell-specification sequence. Certainly, additional investigations will be needed to test this possibility.

The neocortical PVE of other species, notably primates, shows both a lengthening of cell cycle parameters (Kornack and Rakic, 1998) and spatial gradients of expression of bHLH and homeobox transcription factors (Donoghue and Rakic, 1999). The time taken to generate corresponding laminar neuron classes as a proportion of the total neurogenetic interval is uniformly scaled across a wide range of species from rodents to primates (Caviness et al., 1995). Moreover, variations in $\mathrm{T}_{\mathrm{G} 1}$ and/or gradients of transcription factors are known to be characteristic of the proliferative epithelium in other regions of the developing CNS [e.g., the retina (Alexiades and Cepko, 1996; O'Leary and Nakagawa, 2002; Rachel et al., 2002; Schuurmans and Guillemot, 2002; Grove and Fukuchi-Shimogori, 2003)]. Thus, it is plausible that the ability of PVE cells to know their position by monitoring simultaneously $\mathrm{T}_{\mathrm{Gl}}$ and gradient expressions of molecules and then to coordinate this positional information with transcriptional mechanisms related to specification of cell fates and numbers is a general feature of the developing CNS. In essence, the positional information provided by the navigation hypothesis offers a potentially powerful mechanism for the proliferating lineages to "anticipate" the needs of the region that they are destined to produce.

\section{References}

Alexiades MR, Cepko C (1996) Quantitative analysis of proliferation and cell cycle length during development of the rat retina. Dev Dyn 205:293-307.

Boulder Committee (1970) Embryonic vertebrate nervous system: revised terminology. Anat Rec 166:257-262.

Bulchand S, Grove EA, Porter FD, Tole S (2001) LIM-homeodomain gene Lhx2 regulates the formation of the cortical hem. Mech Dev 100:165-175.

Caviness V, Takahashi T, Nowakowski R (1995) Numbers, time and neocortical neuronogenesis: a general developmental and evolutionary model. Trends Neurosci 18:379-383.

Caviness V, Takahashi T, Nowakowski R (2000) Neuronogenesis and the early events of neocortical histogenesis. In: Development of the neocortex (Goffinet A, Rakic P, eds), pp 107-143. Berlin: Springer.

Caviness V, Bhide P, Nowakowski R (2007) Histogenetic processes leading to the laminated neocortex: migration only a part of the story. Dev Neurosci, in press.

Caviness Jr VS, Goto T, Tarui T, Takahashi T, Bhide PG, Nowakowski RS (2003) Cell output, cell cycle duration and neuronal specification: a model of integrated mechanisms of the neocortical proliferative process. Cereb Cortex 13:592-598.

Cunningham D (1985) The brain of the microcephalic idiot. London: The Royal Dublin Society, Williams and Norgate.

Donoghue MJ, Rakic P (1999) Molecular gradients and compartments in the embryonic primate cerebral cortex. Cereb Cortex 9:586-600.

Grove EA, Fukuchi-Shimogori T (2003) Generating the cerebral cortical area map. Annu Rev Neurosci 26:355-380.

Hammarberg C (1895) Studien ueber Klinik und Pathologie der Idiotie nebst Untersuchungen ueber Die normale Anatomie der Hirnrinde. Uppsala, Sweden: Druck der Akademischen Buchdruckerei.

Jessell TM, Melton DA (1992) Diffusable factors in vertebrate embryonic induction. Cell 68:257-270.

Kerner JA, Standaert DG, Penney Jr JB, Young AB, Landwehrmeyer GB (1998) Simultaneous isotopic and nonisotopic in situ hybridization histochemistry with cRNA probes. Brain Res Brain Res Protoc 3:22-32.

Kornack DR, Rakic P (1998) Changes in cell-cycle kinetics during the development and evolution of primate neocortex. Proc Natl Acad Sci USA 95:1242-1246.

Melton D (1991) Pattern formation during animal development. Science 252:234-241.

Mitsuhashi T, Aoki Y, Eksioglu YZ, Takahashi T, Bhide PG, Reeves SA, Caviness VS, Jr (2001) Overexpression of p27Kipl lengthens the G1 phase in a mouse model that targets inducible gene expression to central nervous system progenitor cells. Proc Natl Acad Sci USA 98:6435-6440.

Miyama S, Takahashi T, Nowakowski RS, Caviness VS, Jr (1997) A gradient in the duration of the G1 phase in the murine neocortical proliferative epithelium. Cereb Cortex 7:678-689.

Monuki ES, Porter FD, Walsh CA (2001) Patterning of the dorsal telencephalon and cerebral cortex by a roof plate-Lhx2 pathway. Neuron 32:591-604.

Murata K, Hattori M, Hirai N, Shinozuka Y, Hirata H, Kageyama R, Sakai T, 
Minato N (2005) Hes1 directly controls cell proliferation through the transcriptional repression of p27Kip1. Mol Cell Biol 25:4262-4271.

Nakagawa Y, O'Leary DD (2001) Combinatorial expression patterns of LIM-homeodomain and other regulatory genes parcellate developing thalamus. J Neurosci 21:2711-2725.

Nakagawa Y, Johnson JE, O’Leary DD (1999) Graded and areal expression patterns of regulatory genes and cadherins in embryonic neocortex independent of thalamocortical input. J Neurosci 19:10877-10885.

Novak B, Tyson JJ (2003) Modelling the controls of the eukaryotic cell cycle. Biochem Soc Trans 31:1526-1529.

Novak B, Tyson JJ (2004) A model for restriction point control of the mammalian cell cycle. J Theor Biol 230:563-579.

Nowakowski RS, Caviness Jr VS, Takahashi T, Hayes NL (2002) Population dynamics during cell proliferation and neuronogenesis in the developing murine neocortex. Results Probl Cell Differ 39:1-25.

O'Leary DD, Nakagawa Y (2002) Patterning centers, regulatory genes and extrinsic mechanisms controlling arealization of the neocortex. Curr Opin Neurobiol 12:14-25.

Porter FD, Drago J, Xu Y, Cheema SS, Wassif C, Huang SP, Lee E, Grinberg A, Massalas JS, Bodine D, Alt F, Westphal H (1997) Lhx2, a LIM homeobox gene, is required for eye, forebrain, and definitive erythrocyte development. Development 124:2935-2944.

Rachel RA, Dolen G, Hayes NL, Lu A, Erskine L, Nowakowski RS, Mason CA (2002) Spatiotemporal features of early neuronogenesis differ in wildtype and albino mouse retina. J Neurosci 22:4249-4263.

Reber M, Burrola P, Lemke G (2004) A relative signalling model for the formation of a topographic neural map. Nature 431:847-853.

Roy K, Kuznicki K, Wu Q, Sun Z, Bock D, Schutz G, Vranich N, Monaghan AP (2004) The Tlx gene regulates the timing of neurogenesis in the cortex. J Neurosci 24:8333-8345.
Sarmento LM, Huang H, Limon A, Gordon W, Fernandes J, Tavares MJ, Miele L, Cardoso AA, Classon M, Carlesso N (2005) Notch1 modulates timing of G1-S progression by inducing SKP2 transcription and p27 Kip1 degradation. J Exp Med 202:157-168.

Schuurmans C, Guillemot F (2002) Molecular mechanisms underlying cell fate specification in the developing telencephalon. Curr Opin Neurobiol 12:26-34.

Sobel D (1995) Longitude. New York: Walker and Company.

Takahashi T, Nowakowski RS, Caviness Jr VS (1995) The cell cycle of the pseudostratified ventricular epithelium of the embryonic murine cerebral wall. J Neurosci 15:6046-6057.

Takahashi T, Nowakowski RS, Caviness Jr VS (1996) The leaving or Q fraction of the murine cerebral proliferative epithelium: a general model of neocortical neuronogenesis. J Neurosci 16:6183-6196.

Takahashi T, Goto T, Miyama S, Nowakowski RS, Caviness Jr VS (1999) Sequence of neuron origin and neocortical laminar fate: relation to cell cycle of origin in the developing murine cerebral wall. J Neurosci 19:10357-10371.

Tarui T, Takahashi T, Nowakowski RS, Hayes NL, Bhide PG, Caviness VS (2005) Overexpression of p27 Kip 1, probability of cell cycle exit, and laminar destination of neocortical neurons. Cereb Cortex 15:1343-1355.

Wolpert L, Beddington R, Brockes J, Jessell T, Lawrence P, Meyerowitz E (1998) Principles of development. London: Oxford UP.

Yoon K, Gaiano N (2005) Notch signaling in the mammalian central nervous system: insights from mouse mutants. Nat Neurosci 8:709-715.

Yun K, Mantani A, Garel S, Rubenstein J, Israel MA (2004) Id4 regulates neural progenitor proliferation and differentiation in vivo. Development 131:5441-5448.

Zetterberg A, Larsson O, Wiman KG (1995) What is the restriction point? Curr Opin Cell Biol 7:835-842. 